\title{
PPI-Delayed Diagnosis of Gastrinoma: Oncologic Victim of Pharmacologic Success
}

\author{
Hilda Wong • Thomas Yau • Pierre Chan • \\ Irene O. L. Ng • Gavin Chan • Peter Hui • W. L. Law • \\ C. M. Lo $\cdot$ Antony J. Hedley $\cdot$ Richard J. Epstein
}

Received: 18 April 2009 /Accepted: 23 July 2009 / Published online: 20 August 2009

(C) Arányi Lajos Foundation 2009

\begin{abstract}
Functional neuroendocrine tumors are often lowgrade malignant neoplasms that can be cured by surgery if detected early, and such detection may in turn be accelerated by the recognition of neuropeptide hypersecretion syndromes. Uniquely, however, relief of peptic symptoms induced by hypergastrinemia is now available from acid-suppressive drugs such as proton-pump inhibitors (PPIs). Here we describe a clinical case in which time to diagnosis from the onset of peptic symptoms was delayed more than 10 years, in part reflecting symptom masking by continuous prescription of the PPI omeprazole. We propose diagnostic criteria for this under-recognized new clinical syndrome, and recommend that physicians routinely measure serum gastrin levels in persistent cases of PPI-dependent dyspepsia unassociated with H. pylori.
\end{abstract}

H. Wong $\cdot$ T. Yau $\cdot$ R. J. Epstein

Division of Hematology/Oncology,

Department of Medicine, Queen Mary Hospital,

Hong Kong, Hong Kong

P. Chan

Division of Gastroenterology,

Department of Medicine, Queen Mary Hospital,

Hong Kong, Hong Kong

\section{O. $\mathrm{L} . \mathrm{Ng} \cdot \mathrm{G}$. Chan}

Department of Pathology, Queen Mary Hospital,

The University of Hong Kong,

Hong Kong, Hong Kong

P. Hui

Department of Radiology, Queen Mary Hospital,

The University of Hong Kong,

Hong Kong, Hong Kong
Keywords Neuroendocrine carcinoma $\cdot$ Pancreatic tumor Zollinger-Ellison syndrome · Gastrin · Omeprazole

\author{
Abbreviations \\ PNET pancreatic neuroendocrine tumour \\ PPI proton pump inhibitor \\ ZES Zollinger-Ellison syndrome
}

\section{Background}

Pancreatic neuroendocrine tumors (PNETs) are generally characterised by slow onset of non-specific symptoms and consequent delay in diagnosis. With specific reference to gastrinomas, Ellison \& Sparks showed in a prospective

W. L. Law $\cdot$ C. M. Lo

Department of Surgery, Queen Mary Hospital,

The University of Hong Kong,

Hong Kong, Hong Kong

A. J. Hedley

Department of Community Medicine,

The University of Hong Kong,

Hong Kong, Hong Kong

R. J. Epstein $(\square)$

Department of Medicine, Queen Mary Hospital,

Professorial Block, 102 Pokfulam Road,

Hong Kong, Hong Kong

e-mail: repstein@hku.hk

Present address:

R. J. Epstein

Department of Medical Oncology,

St. Vincent's Hospital,

Victoria St., Darlinghurst,

Sydney 2010, Australia

e-mail: repstein@stvincents.com.au 
study that the era of proton pump inhibitors (PPIs) although highly effective symptomatic therapy for the neuroendocrine symptoms of gastrinoma-has been associated with upstaging of malignant disease at diagnosis [1]. A retrospective analysis reached the same conclusion based on correlations of case referral patterns over time [2]; however, the expected secondary incidence rise following diagnostic delay was not confirmed, casting doubt on a causal interpretation. Confusingly, in the largest clinical analysis of Zollinger-Ellison syndrome ever published, this same group reported no change in age of onset, delay in diagnosis (about 5 years) or frequency of complications following introduction of PPIs [3]. Since these negative conclusions could plausibly have been confounded by concurrent improvements in diagnostic technology and/or physician awareness, we present here an instructive case of malignant gastrinoma that clearly illustrates how a suboptimal index of suspicion can delay diagnosis to more than 10 years after the first presentation of reflux symptoms managed with PPIs.

\section{Case Presentation}

A 58-year-old Caucasian male presented in 2000 with a long history of gastroesophageal reflux symptoms since the 1990s; his past history was notable for hypertension and appendicectomy, and his family history was negative. Upper endoscopy showed gastroesophageal reflux disease, and a segment of Barrett's esophagus was confirmed by biopsy. $\mathrm{He}$ was treated initially with the H2-receptor antagonist ranitidine, but this was soon changed to the PPI omeprazole (20 mg/day) due to inadequate pain control. His symptoms briskly recurred on discontinuing omeprazole, leading to indefinite continuation of this medication as long-term maintenance. Upper endoscopy was repeated every 2 years for monitoring. In 2001 the symptom complex evolved to include recurrent episodic abdominal pain of cramping nature associated with retching and prominent borborygmi. Diarrhea was not a feature; instead, constipation was reported, with abdominal X-rays showing subacute intestinal obstruction. Colonoscopy was unremarkable. The patient was followed up in a primary healthcare setting.

In March 2004 he presented again, this time with subacute right upper quadrant pain. A contrast magnetic resonance imaging (MRI) scan of the hepatobiliary system revealed no abnormality apart from two small liver nodules, measuring $11 \mathrm{~mm}$ in segment VI, and $20 \mathrm{~mm}$ in segment VII, respectively, which were hypointense on plain T1weighted images, and hyperintense with a 'light-bulb' appearance on plain T2-weighted images (Fig. 1a), with peripheral enhancement and central filling-in on postcontrast $\mathrm{T} 1$-weighted images. The radiological features
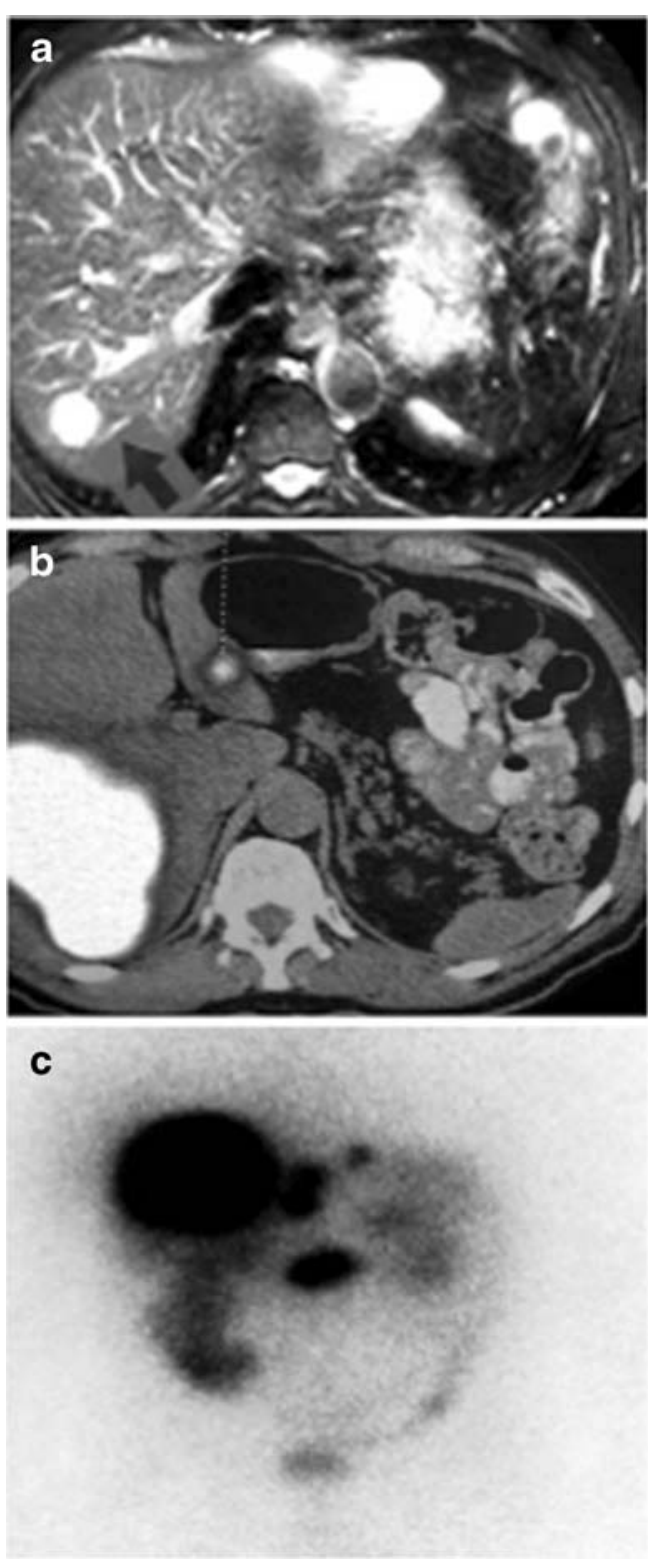

Fig. 1 a MRI liver in 2004 showing one of the lesions which was hyperintense with a typical 'light-bulb' appearance on plain T2weighted images. b FDG-PET scan showing uptake in the liver and pancreatic lesions with regional lymph node involvement. c OctreoScan confirming metastatic pancreatic neuroendocrine tumor

were deemed typical of hemangiomas, so no biopsy was advised. Maintenance omeprazole was increased to $20 \mathrm{mg}$ twice-daily, and the pain resolved.

In 2007 the patient underwent a further reassessment for recurrent episodic abdominal discomfort. Upper endoscopy at this time confirmed persistent esophagitis with no frank ulceration; the previous changes of Barrett's esophagus had resolved on long-term PPI. In January 2008 he was admitted to a tertiary referral centre for an acute exacerbation of abdominal pain. Physical examination revealed a febrile patient with mild tenderness over the right lower 
quadrant. Routine blood tests revealed a raised white cell count of $11.4 \times 10^{9} / \mathrm{L}$ (normal: $4.4-10.1 \times 10^{9} / \mathrm{L}$ ) with neutrophilia, normal renal function and electrolytes including calcium; mildly elevated serum alkaline phosphatase of 123 $\mathrm{U} / \mathrm{L}$ (normal: $42-110 \mathrm{U} / \mathrm{L}$ ) and aspartate transaminase of $55 \mathrm{U} / \mathrm{L}$ (normal: 15-38 U/L), and normal bilirubin and clotting profile. Plain abdominal X-ray revealed a fecalloaded colon with prominent small bowel loops. Computed tomography $(\mathrm{CT})$ of the abdomen and pelvis was performed to exclude diverticulitis; this revealed a $10 \mathrm{~cm}$ heterogenous mass spanning segments V, VI, VII and VIII of the right lobe of the liver, a second intrahepatic mass occupying segments VI and VII adjacent to it, and a pancreatic mass at the uncinate process with regional mesenteric lymphadenopathy. Tumor markers including alpha-fetoprotein (AFP), prostate specific antigen (PSA) and Ca 19.9 were normal; carcinoembryonic antigen (CEA) was slightly raised to $7.4 \mathrm{ng} / \mathrm{ml}$ (normal: $<5 \mathrm{ng} / \mathrm{ml}$ ). The patient then underwent positron emission tomography/computed tomography (PET-CT) with 18-fluorodeoxyglucose (FDG), which showed hypermetabolic lesions in the liver, pancreas and mesenteric lymph nodes (Fig. 1b). Ultrasound-guided biopsy of the liver mass confirmed a low-grade neuroendocrine tumor; immunohistochemistry for synaptophysin, chromogranin and CD56 was positive. Other markers, including inhibin, c-kit, AFP, HEPA, CK7 and CK20, were negative.

Further neuroendocrine workup was performed after stopping PPI for two weeks. Fasting serum gastrin remained elevated $(240 \mathrm{pg} / \mathrm{ml}$; normal $<113 \mathrm{pg} / \mathrm{ml})$, with less marked elevations of chromogranin A $(247 \mathrm{ng} / \mathrm{ml}$; normal $<160 \mathrm{ng} / \mathrm{ml})$, and somatostatin $(25 \mathrm{pg} / \mathrm{ml}$; normal $<22 \mathrm{pg} / \mathrm{ml}$ ). Fasting plasma glucose, insulin, C-peptide and glucagon, and urinary vanillylmandelic acid (VMA) and catecholamines were normal, but 24-hour urinary 5-hydroxyindoleacetic acid was raised. Gastric acid output studies were not performed. Somatostatin receptor imaging (OctreoScan) showed intense uptake in the uncinate process of the pancreas, and in the right lobe, segment II, III and caudate lobe of liver, compatible with a neuroendocrine tumor rich in somatostatin receptors (Fig. 1c).

In March 2008 the patient underwent the first of two laparotomies. Because of the symptomatic nature of the large hepatic metastases, partial hepatectomy was performed first. A $10 \mathrm{~cm}$ tumor was found in the right lobe of the liver, and smaller tumors were found in segment II and III; right hepatectomy was carried out with wedge resection of the segment II lesion, radiofrequency ablation of the segment III lesion, followed by wedge resection of segment III. Histopathologic examination confirmed a metastatic well-differentiated neuroendocrine tumor of low grade malignancy. Expression of the proliferation marker
MIB-1 (Ki-67) was less than 5\%. As expected, immunohistochemical studies were positive for chromogranin, synaptophysin, CD56, serotonin and gastrin.

Whipple's pancreatectomy and anastomosis was performed six weeks later, and a $5 \mathrm{~cm}$ uncinate process primary pancreatic islet-cell tumor removed. Pathology showed a well-differentiated pancreatic neuroendocrine tumor with lymphovascular permeation and regional lymph node metastases; immunohistochemical staining again confirmed the positivity of neuroendocrine markers (Fig. 2). Based on the symptom diathesis, biochemical analyses and histopathological findings, a clinical diagnosis of malignant pancreatic gastrinoma was made. Post-surgical follow-up showed normal gastrin and chromogranin levels. Urinary 5-HIAA level was much reduced compared to baseline, though still marginally raised. Octreoscan showed uptake at the liver resection margin, but no definitive lesion was confirmed by MRI. One year later, the patient remained asymptomatic.

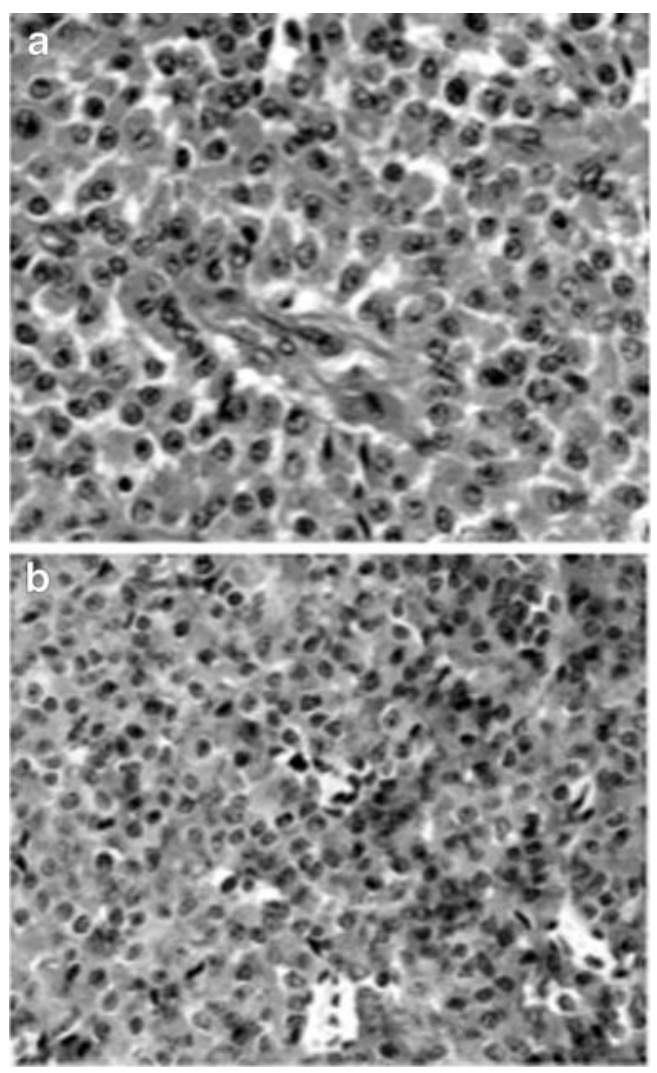

Fig. 2 a Microscopic examination of the pancreatic primary, showing well-differentiated neuroendocrine tumor with regional lymph node involvement. The tumour cells are arranged in sheets with vascular stroma. They have mild nuclear pleomorphism, abundant eosinophilic cytoplasm and eccentric nuclei. Regional lymph node involvement is present (not shown). Original magnification $x 400$. b Immunohistochemical staining of the pancreatic primary, showing positive neuroendocrine marker synaptophysin. Original magnification $x 400$ 


\section{Discussion}

The earliest presenting symptoms of functional PNETs depend on the consequences of their hormonal output. In gastrinomas the clinical presentation depends on the effects of constitutive gastrin production-mainly, in historical studies, gastric acid hypersecretion - and that of any other gut neuropeptide(s) released. Although the classical Zollinger-Ellison syndrome (ZES) described multiple and/or ectopic peptic ulcers $[4,5]$, a recent study showed that the usual presenting symptoms of gastrinomas now feature abdominal pain, diarrhea, or both in $75 \%, 73 \%$ and $55 \%$ of patients respectively, with heartburn also reported in $44 \%$ [3]. Moreover, even prior to the advent of PPI therapy, up to $25 \%$ of malignant gastrinoma patients have no demonstrable peptic ulceration [6].

Up to a quarter of all gastrinomas are associated with multiple endocrine neoplasia type I (MEN 1), which is inherited in an autosomal dominant manner. Although gastrinoma is the most common pancreatic islet cell tumour type in MEN 1, the most crucial component and usual initial presentation of the syndrome tends to be primary hyperparathyroidism, present in over $90 \%$ of MEN 1 patients [7]. Moreover, islet cell tumours in MEN 1 are typically multiple. In view of normocalcemia, solitary nature of the gastrinoma, lack of associated clinical features and negative family history, the current case was considered a sporadic occurence.

We submit that the present case illustrates the features of a new clinical syndrome of 'PPI-masked gastrinoma'. First, the patient's symptoms are predominantly not those of hyperacidity, but rather those of hypergastrinemia - recurrent abdominal pain, gastrointestinal hypermotility, borborygmi, and gallbladder contraction. In contrast to historical ZES descriptions, effective PPI-dependent suppression of massive gastric acid hypersecretion should reduce both peptic ulceration and diarrhea $[8,9]$, consistent with the present case in which the only symptom of low gastric $\mathrm{pH}$ was occasional heartburn. Indeed, omeprazole has long been recognized to be a more tolerable and effective therapy for gastrinoma symptoms than is the gastrininhibitory somatostatin analog, octreotide [10]. Although we would predict prominent gastric mucosal rugosity without ulceration to be another correlate of this syndrome [11], we have not documented this.

Second, the delay in diagnosis of this case from the time of symptom onset is typical of PPI-masked gastrinoma. This iatrogenic complication - in which, ironically, an established ZES supportive therapy [12,13] is used for symptom relief without knowledge of the etiologic diagnosis, culminating in eventual diagnosis of more advanced disease with shorter average 5-year survival, perhaps reflecting a lead time effect-should be a matter of serious professional concern. In the present case the diagnosis of gastrinoma was masked by PPI-dependent reversion of hyperacidity, leaving only the nonspecific syndrome of intermittent abdominal pain and borborygmi. Indeed, so minimally symptomatic was the PPI-treated patient for several years that the early radiologic detection of liver metastases was misinterpreted, and the final diagnosis of malignancy was only made in response to painful liver capsular distension by massive tumor progression 4 years later. Since half of all ZES presentations occur in the absence of detectable liver metastases [14], early recognition of hypergastrinemic symptoms prior to carcinomatosis would seem the only feasible pathway to cure.

Third, the ease of misdiagnosing PPI-masked gastrinoma as an atypical carcinoid syndrome or even as a nonfunctional metastatic PNET is well illustrated by the present case, providing an explanation for the recent observation by Corleto et al of declining rates of gastrinoma diagnosis associated with rising referral rates for other PNETs. Multiple neuropeptide hypersecretion is often seen in neuroendocrine cancer [15]; this includes that of serotonin production by gastrinomas [16], as well as that of hypergastrinemia occurring in association with carcinoids [17]. It follows from these observations that the heterogeneous clinical presentations of neuroendocrine tumors could well be explained in part by unique multi-neuropeptide signatures of individual tumors. We note that a worse prognosis has been associated with secretion of multiple neuropeptides by sporadic PNETs [18], further emphasizing the hazards of late diagnosis occurring as a result of PPImasked symptoms.

The suboptimal sensitivity and specificity in diagnosing pancreatic neuroendocrine tumors by conventional imaging techniques also adds to the diagnostic dilemma. For example, the sensitivity of MRI in detecting these tumors is reported to vary between $29 \%$ to $57 \%$ only [19-21]. MRI visualization of islet cell tumors, which have a rich arterial blood supply [21], depends on their hypervascularity relative to the surrounding normal pancreatic tissue [17], but it is difficult to distinguish them from other vascular lesions including hemangiomas. In the present case, tumors already present on MRI liver in 2004 were misinterpreted as benign hemangiomas (Fig. 1a). Curiously, FDG-PET is reputed to be less useful in well-differentiated slowgrowing neuroendocrine tumors $[18,19]$ but was of value in our case; newer positron-emitter radiopharmaceuticals including ${ }^{11} \mathrm{C}$ 5HTP and ${ }^{11} \mathrm{C}$ L-DOPA appear more sensitive [20]. Somatostatin receptor scintigraphy is superior to ultrasound, CT, MRI, and FDG-PET in terms of both sensitivity and specificity [22], though ${ }^{11} \mathrm{C}$ 5HTP-PET may prove even more sensitive.

In summary, the present case supports other clinical observations [23, 24] and series [1,25] attesting to the evolution of a new iatrogenic syndrome caused by PPI- 
dependent suppression of peptic symptoms. We propose a diagnostic triad for this novel syndrome comprising (i) nonpeptic symptoms in hypergastrinemic patients receiving maintenance PPI, (ii) late-stage presentation of metastatic PNET, and (iii) diagnostic confusion with non-gastrinoma PNETs. Despite the negative finding by Roy et al with respect to diagnostic delay in PPI-treated gastrinomas [3], we submit that this emerging entity is indeed valid, and encourage a higher level of awareness by clinicians using PPIs to treat chronic non- $H$. pylori-associated hypergastrinemic dyspepsia, especially in middle-aged patients with unremitting, albeit non-specific, gastrointestinal symptoms.

Acknowledgements We thank Professor Kjell Oberg of Karolinska Institute for helpful discussions about this case.

Consent Written informed consent was obtained from the patient for publication of this case report and any accompanying images. A copy is available for review by the Editor-in-Chief of this journal.

Competing interests The authors declare that they have no competing interests.

Authors' contributions HW and RE (guarantor) drafted the report. TY and PC participated in analysis. IN and GC did immunohistochemistry. PH reported imaging. All authors conceived, read and revised the manuscipt.

\section{References}

1. Ellison EC, Sparks J (2003) Zollinger-Ellison syndrome in the era of effective acid suppression: are we unknowingly growing tumors? Am J Surg 186:245-8

2. Corleto VD, Annibale B, Gibril F et al (2001) Does the widespread use of proton pump inhibitors mask, complicate and/ or delay the diagnosis of Zollinger-Ellison syndrome? Aliment Pharmacol Ther 15:1555-61

3. Roy PK, Venzon DJ, Shojamanesh H et al (2000) ZollingerEllison syndrome. Clinical presentation in 261 patients. Medicine (Baltimore) 79:379-411

4. Ellison EH, Wilson SD (1964) The Zollinger-Ellison syndrome: re-appraisal and evaluation of 260 registered cases. Ann Surg 160:512-30

5. Zollinger RM, Ellison EC, O'Dorisio TM et al (1984) Thirty years' experience with gastrinoma. World J Surg 8:427-35

6. Jensen RT, Gardner JD, Raufman JP et al (1983) ZollingerEllison syndrome: current concepts and management. Ann Intern Med 98:59-75

7. DeVita VT, Lawrence TS, Rosenberg SA (2008) Cancer: principles of oncology. 8th Ed. Lippincott Williams \& Welkins.
8. Blonski WC, Katzka DA, Lichtenstein GR et al (2005) Idiopathic gastric acid hypersecretion presenting as a diarrheal disorder and mimicking both Zollinger-Ellison syndrome and Crohn's disease. Eur J Gastroenterol Hepatol 17:441-4

9. Roy PK, Venzon DJ, Feigenbaum KM et al (2001) Gastric secretion in Zollinger-Ellison syndrome. Correlation with clinical expression, tumor extent and role in diagnosis - - a prospective $\mathrm{NIH}$ study of 235 patients and a review of 984 cases in the literature. Medicine (Baltimore) 80:189-222

10. Maton PN (1993) Use of octreotide acetate for control of symptoms in patients with islet cell tumors. World J Surg 17:504-10

11. Fukui T, Nishio A, Okazaki K et al (2006) Gastric mucosal hyperplasia via upregulation of gastrin induced by persistent activation of gastric innate immunity in major histocompatibility complex class II deficient mice. Gut 55:607-15

12. Metz DC, Comer GM, Soffer E et al (2006) Three-year oral pantoprazole administration is effective for patients with Zollinger-Ellison syndrome and other hypersecretory conditions. Aliment Pharmacol Ther 23:437-44

13. Metz DC, Sostek MB, Ruszniewski P et al (2007) Effects of esomeprazole on acid output in patients with Zollinger-Ellison syndrome or idiopathic gastric acid hypersecretion. Am J Gastroenterol 102:2648-54

14. Banasch M, Schmitz F (2007) Diagnosis and treatment of gastrinoma in the era of proton pump inhibitors. Wien Klin Wochenschr 119:573-8

15. Lodish MB, Powell AC, Abu-Asab M et al (2008) Insulinoma and gastrinoma syndromes from a single intrapancreatic neuroendocrine tumor. J Clin Endocrinol Metab 93:1123-8

16. Gurevich L, Kazantseva I, Isakov VA et al (2003) The analysis of immunophenotype of gastrin-producing tumors of the pancreas and gastrointestinal tract. Cancer 98:1967-76

17. Bamba T, Kosugi S, Kanda $\mathrm{T}$ et al (2007) Multiple carcinoids in the duodenum, pancreas and stomach accompanied with type A gastritis: a case report. World J Gastroenterol 13:2247-9

18. Ardill JE, Erikkson B (2003) The importance of the measurement of circulating markers in patients with neuroendocrine tumours of the pancreas and gut. Endocr Relat Cancer 10:459-62

19. Gibril F, Reynolds JC, Doppman JL et al (1996) Somatostatin receptor scintigraphy: its sensitivity compared with that of other imaging methods in detecting primary and metastatic gastrinomas A prospective study. Ann Intern Med 125:26-34

20. Zimmer T, Stolzel U, Bader M et al (1996) Endoscopic ultrasonography and somatostatin receptor scintigraphy in the preoperative localisation of insulinomas and gastrinomas. Gut 39:562-8

21. Alexander HR, Fraker DL, Norton JA et al (1998) Prospective study of somatostatin receptor scintigraphy and its effect on operative outcome in patients with Zollinger-Ellison syndrome. Ann Surg 228:228-38

22. Thompson NW, Eckhauser FE, Vinik AI et al (1984) Cystic neuroendocrine neoplasms of the pancreas and liver. Ann Surg 199:158-64

23. van Santen S, van Soest EJ, Busch OR et al (2007) A woman with shock as first sign of Zollinger-Ellison syndrome. Ned Tijdschr Geneeskd 151:478-83

24. Tartaglia A, Vezzadini C, Bianchini S et al (2005) Gastrinoma of the stomach: a case report. Int J Gastrointest Cancer 35:211-6

25. Norton JA, Fraker DL, Alexander HR et al (1999) Surgery to cure the Zollinger-Ellison syndrome. N Engl J Med 341:635-44 